\title{
Aortic sutureless bioprosthesis with concomitant Ferrazzi procedure, Coronary revascularization and Neo-fenestrated pericardial reconstruction: report of a case
}

\author{
Faizus Sazzad $^{1 *}$, Vidanaptthirana Chirantha Puwalani ${ }^{1}$, Chang Guohao ${ }^{1}$, Julian Tan $^{2}$, Theodoros Kofidis ${ }^{1}$ \\ From World Society of Cardiothoracic Surgeons 25th Anniversary Congress, Edinburgh \\ Edinburgh, UK. 19-22 September 2015
}

\section{Background/Introduction}

The clinical outcome of sutureless aortic bioprosthesis in concomitant complex heart surgery is still unclear.

\section{Aims/Objectives}

We assessed a patient with large left ventricular aneurysm underwent concomitant procedure.

\section{Method}

We report a case of 66 years Malay gentleman, diagnosed as large $\mathrm{LV}$ aneurysm $(7.8 \times 4.9 \times 8.0 \mathrm{~cm})$ secondary to previous anterior MI, Moderate aortic valve stenosis, Triple vessel coronary disease; underwent LV aneurysmectomy (Ferrazzi procedure) removal of LV thrombus, Sutureless aortic valve replacement (Perceval S), Coronary revascularization and Neo-fenestrated pericardial reconstruction with intra-aortic balloon counter pulsation support. At operation the large LV aneurysm was found at anterior-inferior apical area and was self-limiting leaking at the apical area (Almost ruptured) and was in-folded and got adherent to RV wall. Cardio-pulmonary bypass time was $199 \mathrm{~min}$, and Cross-clamp $146 \mathrm{~min}$.

\section{Results}

Post operatively patient underwent resternotomy for bleeding and chest wash out on post-op day 1.Complicated with Bowel perforation and needed emergency exploratory laparotomy, Descending colon resection, with double barrel stoma creation, subsequently recovered well. Post op TTE showed LVIDD came down to $50 \mathrm{~mm}$ (Preoperative 72) with improved ejection fraction and good functioning sutureless valve.

\section{Discussion/Conclusion}

Duration of surgery in complex concomitant procedure is an influencing factor for successful outcome. Sutureless aortic bioprosthesis reduces the operative length hence its use should be prioritized in such cases.

\section{Consent}

Written informed consent was obtained from the patient for publication of this abstract and any accompanying images. A copy of the written consent is available for review by the Editor of this journal.

\section{Authors' details}

${ }^{1}$ Department of Cardiac Thoracic and Vascular Surgery National University

Hospital, Singapore, Singapore. ${ }^{2}$ Tan Tock Seng Hospital, Singapore, Singapore.

Published: 16 December 2015

doi:10.1186/1749-8090-10-S1-A309

Cite this article as: Sazzad et al:: Aortic sutureless bioprosthesis with concomitant Ferrazzi procedure, Coronary revascularization and Neofenestrated pericardial reconstruction: report of a case. Journal of Cardiothoracic Surgery 2015 10(Suppl 1):A309. 